\title{
Anatomy-Focused Volume Line Integral Convolution for Brain White Matter Visualization
}

\author{
T. Schult \\ Institute for Applied \\ Computer Science \\ Stralsund University of \\ Applied Sciences \\ Zur Schwedenschanze \\ 15 \\ Germany, 18435 \\ Stralsund, MV \\ thomas.c.schult@hochsc \\ hule-stralsund.de
}

\author{
U. Klose \\ and Interventional \\ Neuroradiology \\ University Hospital \\ Tübingen \\ Hoppe-Seyler-Straße 3 \\ Germany, 72076 \\ Tübingen, BW \\ uwe.klose@med.uni- \\ tuebingen.de
}

Department of DiagnosticDepartment of Diagnostic

\author{
T.-K. Hauser \\ and Interventional \\ Neuroradiology \\ University Hospital \\ Tübingen \\ Hoppe-Seyler-Straße 3 \\ Germany, 72076 \\ Tübingen, BW \\ till- \\ karsten.hauser@med.uni \\ -tuebingen.de
}

\author{
H.-H. Ehricke \\ Institute for Applied \\ Computer Science \\ Stralsund University of \\ Applied Sciences \\ Zur Schwedenschanze \\ 15 \\ Germany, 18435 \\ Stralsund, MV \\ hans.ehricke@hochschul
}

e-stralsund.de

\begin{abstract}
3D visualization of volumetric line integral convolution (LIC) datasets has been a field of constant research. So far, most approaches have focused on finding suitable transfer functions and defining appropriate clipping strategies in order to solve the problem of occlusion. In medicine, extensions of the LIC algorithm to diffusion weighted magnetic resonance imaging (dwMRI) have been proposed, allowing highly resolved LIC volumes to be generated. These are used for brain white matter visualization by LIC slice images, depicting fiber structures with good contrast. However, 3D visualization of fiber pathways by volume rendering faces the problem of occlusion of anatomic regions of interest by the dense brain white matter pattern. In this paper, we introduce an anatomyfocused LIC algorithm, which allows specific fiber architectures to be visualized by volume rendering. It uses an anatomical atlas, matched to the dwMRI dataset, during the generation of the LIC noise input pattern. Thus, anatomic fiber structures of interest are emphasized, while surrounding fiber tissue is thinned out and its opacity is modulated. Additionally, we present an adaptation of the orientation-dependent transparency rendering algorithm, which recently has been proposed for fiber streamline visualization, to LIC data. The novel methods are evaluated by application to dwMRI datasets from glioma patients, visualizing fiber structures of interest in the vicinity of the lesion.
\end{abstract}

\section{Keywords}

Volume LIC, Line integral convolution, Anatomical atlas, Visualization, Volume rendering, Diffusion MRI

\section{INTRODUCTION}

Three-dimensional visualization of data from line integral convolution (LIC) is a challenging field in scientific visualization. The major challenges in Volume LIC are occlusion and finding suitable transfer functions. Although streamline tractography has been established as a standard technology, LIC is a promising approach in the visualization of fiber pathways from diffusion weighted magnetic resonance imaging (dwMRI) datasets. However, three-dimensional depiction of LIC data suffers from

Permission to make digital or hard copies of all or part of this work for personal or classroom use is granted without fee provided that copies are not made or distributed for profit or commercial advantage and that copies bear this notice and the full citation on the first page. To copy otherwise, or republish, to post on servers or to redistribute to lists, requires prior specific permission and/or a fee. occlusion and cluttered visualization. Figure 1 presents an example of rendering a dwMRI LIC volume with dense white matter structures. In previous solutions, clipping approaches and sophisticated transfer functions have been proposed to visualize vector fields. In particular, when visualizing dense LIC volumes from dwMRI data, such strategies do not lead to visualizations depicting the threedimensional course of single fiber bundles and lack anatomical context. Furthermore, most of these recent approaches focus on depicting a whole LIC volume after it has been generated.

In this paper, we propose a new approach by emphasizing volumes of interest (VOI) while generating the noise input pattern to the LIC algorithm. This is achieved by the incorporation of an anatomical atlas which is matched to the individual dwMRI dataset, allowing anatomical VOIs to be specified by the user without tedious interactions. Our 
approach is based on the A-Glyph LIC algorithm proposed by Höller et al. [HESK17, HOKG14].

The paper is organized as follows. First, we give an overview of related methods and solutions to the problem of visualizing Volume LIC datasets (section 2 ). In section 3 , we give a detailed description of our approach of anatomy-focused Volume LIC by atlasbased sample placement. In section 4, experimental results from the application of the methods to clinical datasets from glioma patients are presented. We conclude with section 5 by discussing our results and giving an outlook to further algorithmic amendments.

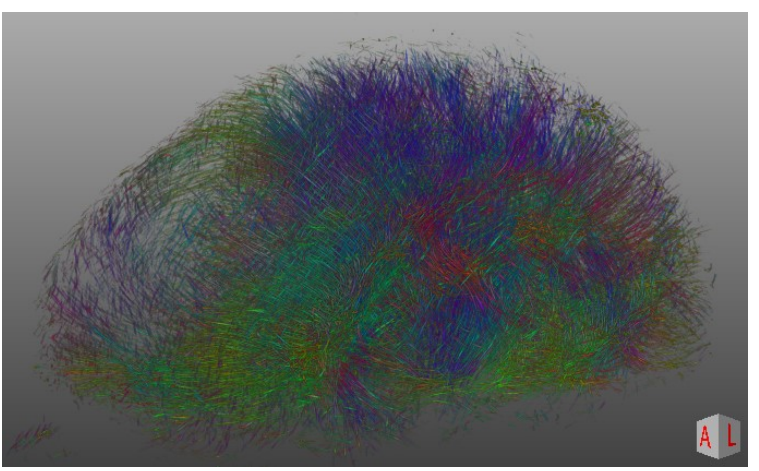

Figure 1. Volume rendering of a Volume LIC dataset. An insight into the inner structures of the brain is occluded. In the front part of the brain, lowered fiber density can be seen.

\section{RECENT SOLUTIONS}

\section{State of the art - Volume LIC}

LIC, originally introduced by Cabral and Leedom [CaLe93], is a texture-based algorithm to visualize vector fields. In its original two-dimensional version, it takes a random noise texture as input and blurs it locally along the given vector field. There are several extensions to the LIC algorithm [LHDV04] that include Volume LIC as well. In scientific literature there are different approaches to visualize Volume LIC data with the aim to give insight into the volumes and to highlight certain characteristics of the underlying data. One area of research focuses on various forms of clipping planes. Schurade et al. [SHSK10] use a Virtual Klinger dissection method with which curved dissection surfaces are generated. These extend locally parallel to user specified fiber bundles and thereby shape a free-form clipping surface.

Peeters et al. [PeVR09] pursue a different approach. They do not calculate a whole LIC volume and depict it, but define a 2D cross section of a heart and carry out a 3D vector field visualization for this "Plane of Interest". In the specified intersection of the plane with the vector field, the $3 \mathrm{D}$ vectors are rendered as $3 \mathrm{D}$ line segments by using a local raycasting approach. In addition, line lightning and shadow computation is performed.

The question of how four-dimensional blood flow patterns can be visualized interactively in vivo is answered by Kainz et al. [KRRS09]. Here, an overview of experiments of how to visualize and measure flow fields in the human vascular system is given and sparse and dense flow visualization algorithms are presented. To visualize the results, a volume raycasting approach is introduced that reintegrates $3 \mathrm{D}$ information into $2 \mathrm{D}$ cutting planes by using velocity directions as plane normal vectors.

Another field of research focuses on the use of transfer functions in generating volume renderings. Tax et al. [TCSV15] do not describe a solution for visualizing Volume LIC, but propose an adaptation of the transparency rendering of fiber trajectories as a function of fiber orientation. This can lead to an enhanced visibility, because pathways that are orientated along a user-specified axis, can be made more transparent. Other approaches render LIC volumes based on 3D Significance Maps [ChFS03, SFCN03]. Significance values are derived from intrinsic properties of the vector field and control the transfer functions for LIC volume rendering by manipulating the opacity of each texel, whereby different regions are highlighted or neglected. Besides the single use of clipping planes and transfer functions, there are several approaches that combine both techniques. Burns et al. [BHWV07] propose feature emphasis and contextual cutaways for multimodal medical visualization. This importance driven approach aims to present embedded data in a way that it is clearly visible along with its spatial relation to the surrounding volumetric material. The specification of importance and ranking of materials within the volume is achieved by augmentation of conventional transfer functions. Additionally, viewdependent cutaway structures are used to cut away occluding materials based on its importance. Similar to the importance driven approach is the employment of cost functions introduced by Auzinger et al. [AMBS13]. A Curved Surface Reformation is proposed to visualize vessel lumen. This method generates a cut surface along a vessel centerline fully in $3 \mathrm{D}$ and handles occlusion by employing a cost function on the cut surface that incorporates the location of individual points to the viewer and to the vessel centerline. Correspondingly necessary cutaways are then generated automatically.

Rezk-Salama et al. extend the use of clipping planes and transfer functions to animated clipping objects that are used for an interactive exploration of Volume LIC based on 3D-Texture Mapping [RHTE99]. Here, LIC calculation is based on sparse noise textures. Through animated clipping objects, an insight into the volume can be given. Additionally, interactive assignment of color and opacity values is used to 
enhance or suppress portions of the data. Liu et al. propose with animated and arbitrarily-oriented cutting planes a similar approach [LiMo05]. Here, timevarying flow volumes are visualized, using volume rendering with magnitude-based transfer functions that show flow structures in individual frames. The rendering method takes two volume datasets as an input. One is the flow texture and the other includes flow magnitude. During visualization only the flow texture is rendered while the flow magnitude is used to define color and opacity mapping in the transfer function.

Highlighting salient features inside volumetrically data is also possible by using short animations during user interactions [SRBG10]. The purpose is to explore the data more efficiently and to aid the user in the detection of unknown features. The method tries to anticipate the region of interest and changes the rendering style to improve the visualization.

The methods listed so far mainly focused on improving the three-dimensional visualization after the volume datasets were generated. However, Interrante et al. present strategies for effectively visualizing 3D flow with Volume LIC [InGr97]. A definition of an appropriate input texture is described, that clarifies the distinct identities and relative depths of the advected texture elements. Regions of interest (ROI) are highlighted in the input and output volumes using "halos". The input texture of the LIC algorithm is sparsely opaque with evenly distributed points that are premultiplied by a function of the velocity of the flow. The halo effect is then achieved by using a second, slightly different input texture which is incorporated in the rendering process. A method that also uses a sparse input texture and produces a halo effect is introduced by Helgeland et al. [HeAn04]. Here, shading is done by limb darkening using a transfer function and performing a two-field visualization. Unlike before, seeding is done by the usage of ROIs, so that seeds are only placed inside these ROIs and the focus of the visualization lies on these regions.

Although the aforementioned methods work well in their respective application areas, they are not directly applicable to Volume LIC data of white matter. Particularly, usage of sophisticated transfer functions to highlight individual fiber bundles is of limited effect, because they do not differ enough in their intensity and change their color in their course. Furthermore, with clipping techniques it is hardly possible to emphasize single fiber bundles and to depict their anatomical context.

\section{METHODS}

\section{A-Glyph LIC}

We base our methods on the A-Glyph LIC approach by Höller et al. [HESK17, HOKG14], which produces high contrast color-coded LIC maps that visualize local anisotropy information and regional fiber architecture of the human brain from dwMRI datasets. Typically, these are acquired by the high-angular resolution diffusion imaging (HARDI) technique. Since the approach has been published before, we give only a short summary here. A-Glyph LIC is an extension of the LIC approach proposed by Cabral and Leedom [CaLe93]. Different to the procedure suggested there, no white noise is used as input texture, because this leads to low-contrast fiber visualizations with poor delineation of fiber pathways. Instead, an anisotropic glyph sample pattern is generated and processed with a multiple kernel LIC algorithm. Since it is the groundwork for the methods described in this paper, we will give a brief explanation in the following. The different steps of the A-Glyph LIC method are illustrated by Figure 2 . The necessary directional vectors to carry out LIC, originate from fiber orientation distributions (FOD) computed by spherical deconvolution from the dwMRI data [TCGC04, ToCC07]. From the FODs the direction vectors of the fibers can be derived by determination of global and local maxima of anisotropic diffusion. The first step of A-Glyph LIC is the generation of a high-resolution input pattern with an isotropic voxel size of $0.1 \mathrm{~mm}$. We use multicylindrical glyph samples derived from the FODs and place them along very short streamlines. The streamlines originate from seeds, which are randomly distributed over the whole volume. Then, a multiple kernel LIC algorithm smoothes the generated highresolution anisotropic glyph input pattern. This is done

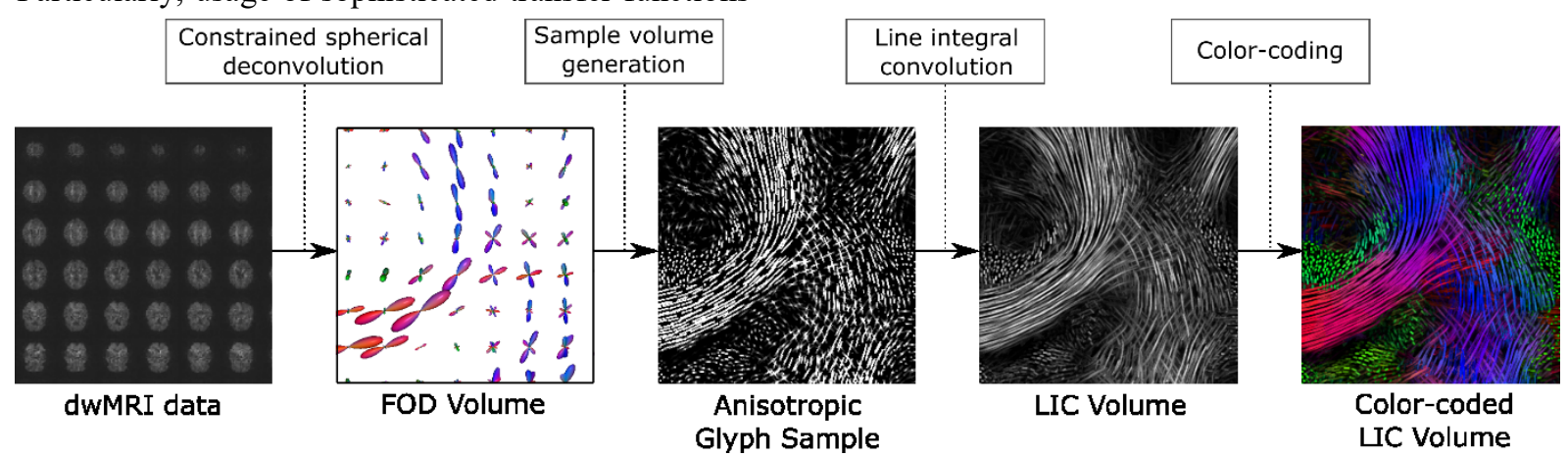

Figure 2. Processing steps of the A-Glyph LIC algorithm. 
not only along a single anisotropy direction, but also along a second local FOD maximum. Thus, crossing and branching fiber pathways can be depicted. In the last step, planar 2D slices are generated from the resulting 3D LIC volume. By directional color-coding fiber orientation in 3D space is depicted [PaPi99].

\section{Proposed Solution}

This paper focusses on Volume LIC data calculated with the A-Glyph LIC algorithm. Originally, the input texture of the convolution process consists of anisotropic glyph samples, placed randomly with the same probability throughout the whole volume. This produces good results for 2D orthogonal slice images, but leads to occlusion in volume renderings (see Figure 1). To overcome this, we introduce an anatomy-driven approach which varies the sample placement probability for different anatomic regions. These regions are defined by usage of an anatomical atlas. The ICBM-DTI-81 atlas is utilized, created from diffusion tensor imaging of 81 healthy volunteers, that segments the white matter of the human brain into fifty areas [MOJJ08]. This atlas is individually registered to the respective dwMRI datasets, using the atlas based segmentation [ChRM94, MCAG06, RoMa05] in the Computational Morphometry Toolkit (CMTK) [Comp18].

During computation of the noise input texture for the LIC algorithm, a VOI is defined by selecting segmented white matter structures of interest. Inside the VOI the probability of placing anisotropic glyph samples is increased from 0.5 percent, what would be the standard probability of the A-Glyph LIC algorithm, to 1.0 percent, whereas in an adjustable environment around the VOI the glyph placement probability is decreased linearly from 1.0 percent to 0.025 percent. This procedure allows the anatomical VOI to be emphasized within the input texture. After application of the LIC algorithm, occlusion by fiber structures outside the VOI is diminished because of a sparser placement of glyph samples outside the specified VOI.
For volume rendering of the anatomy-focused LIC volume we used MeVisLab (MeVis Medical Solutions AG, Bremen, Germany) [Mevi18]. To allow analysis of fiber affection near lesions, we used the integrated surface renderer for 3D display of the tumor surface. In a pre-processing step the tumor is delineated through segmentation with a region growing algorithm. All of the algorithms for tumor delineation and reconstruction are provided by MeVisLab modules.

To enhance 3D visualization, the orientationdependent transparency rendering approach proposed by Tax et al. [TCSV15] was adopted to Volume LIC datasets. The original algorithm of making fibers more transparent along a user-defined axis is based on streamlines, produced by whole-brain tractography. For a single streamline its path through 3D space is known and its overall orientation can easily be computed. Since LIC generated a fiber texture only, the course and overall orientation of a single fiber cannot be determined. However, based on the orientational color-coding scheme, the local orientation of the fiber can be determined for every voxel. The RGB color vector represents a fiber's spatial orientation in $\mathrm{x}-, \mathrm{y}-$ and $\mathrm{z}$-direction. After definition of the viewing direction, all voxels in a conic region around the viewing axis are made more transparent. Thus, fiber structures running orthogonal or nearly orthogonal to the viewing plane are eliminated and no longer occlude fibers of interest. Figure 3 summarizes the processing steps of the anatomy-focused Volume LIC algorithm.

As the computing environment we used a Lenovo ThinkPad S540 laptop computer with an Intel Core i7 processor, 16 GB RAM, and an AMD Radeon HD 8670M GPU with 2 GB VRAM. Computationally expensive routines of the A-GLYPH LIC algorithm were transferred to the graphics hardware using the OpenCL programming language. Thus, highresolution volume LIC datasets could be computed within timeframes of typically approx. 25-30 minutes. Realtime volume rendering by MeVisLab was carried out on the GPU by exploiting the texture mapping hardware.

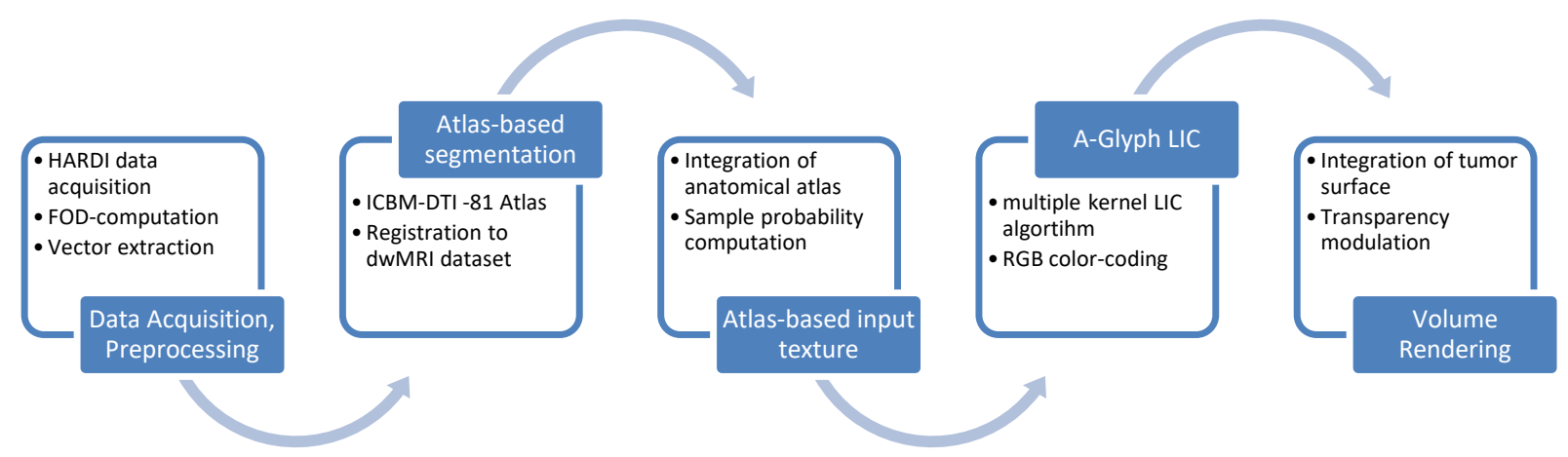

Figure 3. Processing steps of the anatomy-focused Volume LIC algorithm. 


\section{Patient Datasets}

For a preliminary evaluation, two clinical patient datasets with different pathologies were used. The datasets were acquired as part of ongoing research studies approved by the ethics committee of the medical faculty of the Eberhard-Karls-University of Tübingen. Informed-written consent was obtained from the patients or their parents. Patient A is a 54year old woman with a glioblastoma in the right frontal lobe. Patient B is a 15-year old boy with a leftsided brain tumor in the central region close to the primary motor cortex. Both datasets were acquired at the University Hospital Tübingen. Table 1 gives an overview of the acquisition protocols.

\begin{tabular}{|l|l|l|}
\hline & Patient A & Patient B \\
\hline Manufacturer & Siemens & Siemens \\
\hline Scanner & Skyra & Sonata \\
\hline Magnetic field strength [T] & 3.0 & 1.5 \\
\hline Number DWI directions & 64 & 60 \\
\hline B-value [s/mm $\left.{ }^{2}\right]$ & 1000 & 3000 \\
\hline TR/TE [ms] & $6100 / 85$ & $11500 / 122$ \\
\hline Voxel length [mm] & 2.0 & 2.5 \\
\hline
\end{tabular}

Table 1. Diffusion weighted magnetic resonance imaging (dwMRI) acquisition protocols

\section{EXPERIMENTAL RESULTS}

The methods described above were applied to the two clinical datasets. By atlas-based generation of the anisotropic glyph samples the focus was set to clinically relevant regions in the vicinity of the tumor. Figure 4 shows data from Patient A, opposing the original input texture of the A-Glyph LIC algorithm (left) to the atlas-based input texture (right). In this axial view it is clearly noticeable that there are less glyph samples outside the region around the VOI, defined by the corpus callosum (CC). This is because the probability of placing glyph samples outside the VOI was only 2.5 percent of that for placing them inside the VOI. However, there is no smooth transition from inside to outside of the atlas-based VOI. This problem can be solved by smooth adaptation of the placement probability from inside to outside in a continuous manner. The result is demonstrated in Figure 5, showing data from Patient A. Here, the effect of increasing the environment around the VOI is depicted. Figure 5a shows the result of the A-Glyph LIC method with the atlas-based glyph sample shown in Figure 4b. To incorporate the closer neighborhood around the $\mathrm{CC}$, Figure $5 \mathrm{~b}$ expands the VOI by an environment of 5 voxels. Thus, additional anatomical context is displayed. In Figure 5c the environment is expanded even more to 10 voxels. However, this leads to an undesirable occlusion. For this reason, we decided to use an environment of 5 voxels as a default value.

To avoid occlusion by fiber bundles that run along the viewing axis, voxels that represent such fibers are rendered transparent. Figure 6 demonstrates the effect of this occlusion culling technique on an axial view of Patient A. In Figure 6a, fibers that run along the viewing axis are depicted in blue color. They are not rendered transparent. Occlusion is diminished by making fibers transparent that run along the viewing axis within a cone of 45 degrees. As a result, a damaged area of the $\mathrm{CC}$ with lower fiber density becomes visible (marked by yellow arrows).
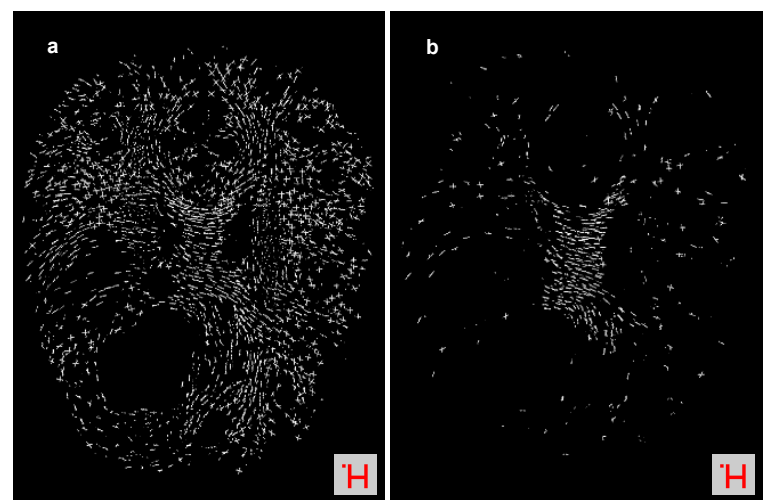

Figure 4. Anisotropic glyph samples for the LIC algorithm in an axial view, generated from the dataset of Patient A. (a) Original input texture, as proposed by Höller et al. (b) Atlas-based input texture with focus on the corpus callosum.

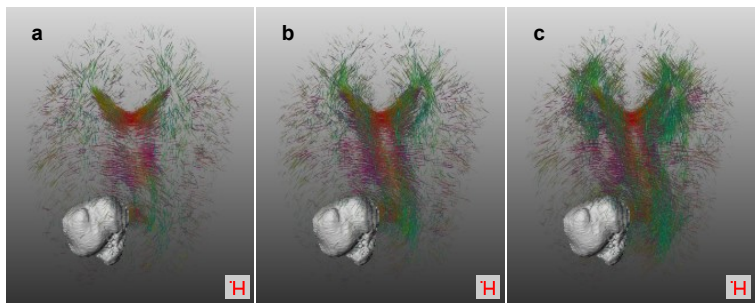

Figure 5. Effect of adjusting the environment around the atlas-based VOI, in which the probability of placing glyph samples is lowered, demonstrated by data from Patient A. (a) No environment, (b) environment of 5 voxels, and (c) environment of 10 voxels.

Through the aforementioned steps a better insight into the inner structure of volume LIC datasets can be given. Figure 7 shows a comparison of volume rendering and anatomy-focused Volume LIC with data from Patient A. Both visualization approaches are combined with surface rendering of the tumor surface. For the atlas-based generation of the anisotropic glyph sample, the corpus callosum was used as VOI, which is a fiber bundle that links both hemispheres of the 


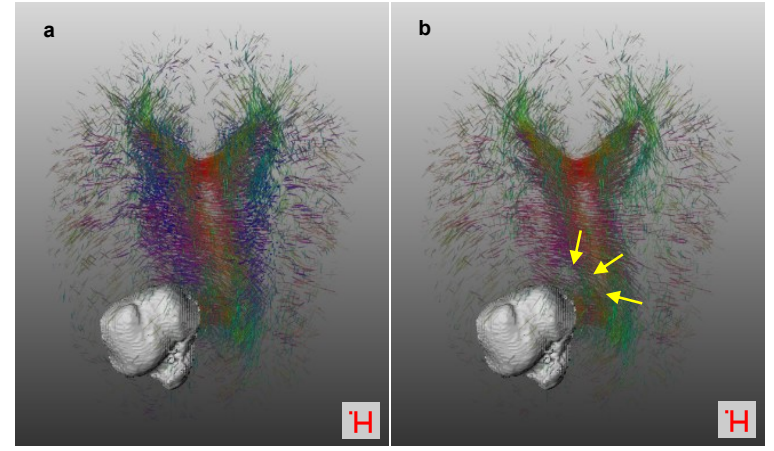

Figure 6. Adaptation of orientation-dependent transparency rendering to anatomy-focused

Volume LIC data of Patient A. (a)Without orientation-dependent transparency rendering.

(b) Transparent rendering of fibers that run along the viewing axis. The yellow arrows mark a region of $\mathrm{CC}$ with reduced fiber density.

brain. It is a central lead structure for neuroradiologists and neurosurgeons for diagnosis and therapy planning. In the example, the $\mathrm{CC}$ is damaged by a tumor and its surrounding edema inside the right frontal lobe. By the anatomical focus inside the input texture of the LIC algorithm and through rendering fibers running from anterior to posterior transparent, the whole $\mathrm{CC}$ and its damaged region become visible (marked by yellow arrowsFigure 8 shows the issue from another perspective. In the volume rendering result of Figure $8 \mathrm{a}$, an insight into inner structures of the brain is prevented by occlusion. The CC is depicted only in part in the fissura longitudinalis. However, by applying the anatomy-focused Volume LIC approach the whole $\mathrm{CC}$ becomes visible and its disruption can be seen in Figure 8b (marked by the yellow arrows). The choice of the anatomical focus to be selected as VOI depends on the pathology of the individual patient dataset. In Patient A, there is a tumor inside the right frontal lobe that damages parts of the $\mathrm{CC}$. Therefore, the atlas-based generation of the anisotropic glyph sample focuses on this region. In the dataset of Patient B, there is a tumor in the left side of the brain that might interfere with the cortico-spinal tract (CST). Neuroradiologists and neurosurgeons often use a left-to-right comparison to analyze pathologies. Therefore, in this case the anatomical focus was on the left and right CST. Figure 9 shows a comparison, similar to that presented by the two previous figures. In Figure 9a the LIC volume is visualized by volume rendering together with the reconstructed tumor surface, located in the left hemisphere. In the image an impairment of a CST is not recognizable. However, Figure $9 b$ indicates a reduced fiber density and disruption of the left branch of the CST in the region, marked by a dotted yellow ellipse, in comparison to the right branch of the CST, marked by a continuous yellow ellipse. To get a better view on the left CST, fibers running from anterior to posterior were rendered transparent.
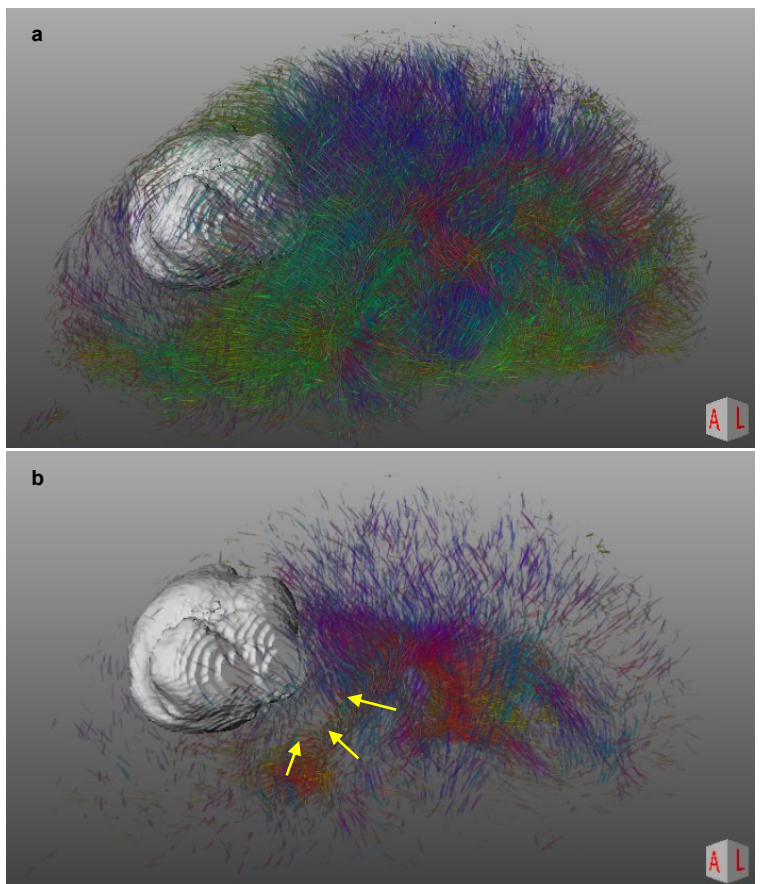

Figure 7. Comparison of (a) volume rendering and (b) anatomy-focused Volume LIC with transparent fibers running from anterior to posterior (Patient $A$ ). On both visualizations the same look-up table has been used and both are combined with the reconstructed tumor surface

(grey). The arrows point to a pathological disruption of the corpus callosum.

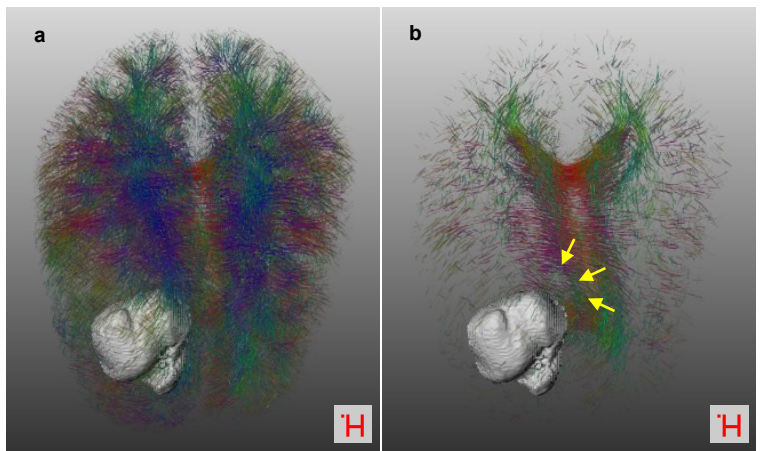

Figure 8. Same comparison as in Figure 7, but with an axial view. (a) Volume rendering with occlusion of inner structures of the brain. (b)

Anatomy-focused visualization of the $\mathrm{CC}$, revealing a disrupted region, marked by yellow arrows. 

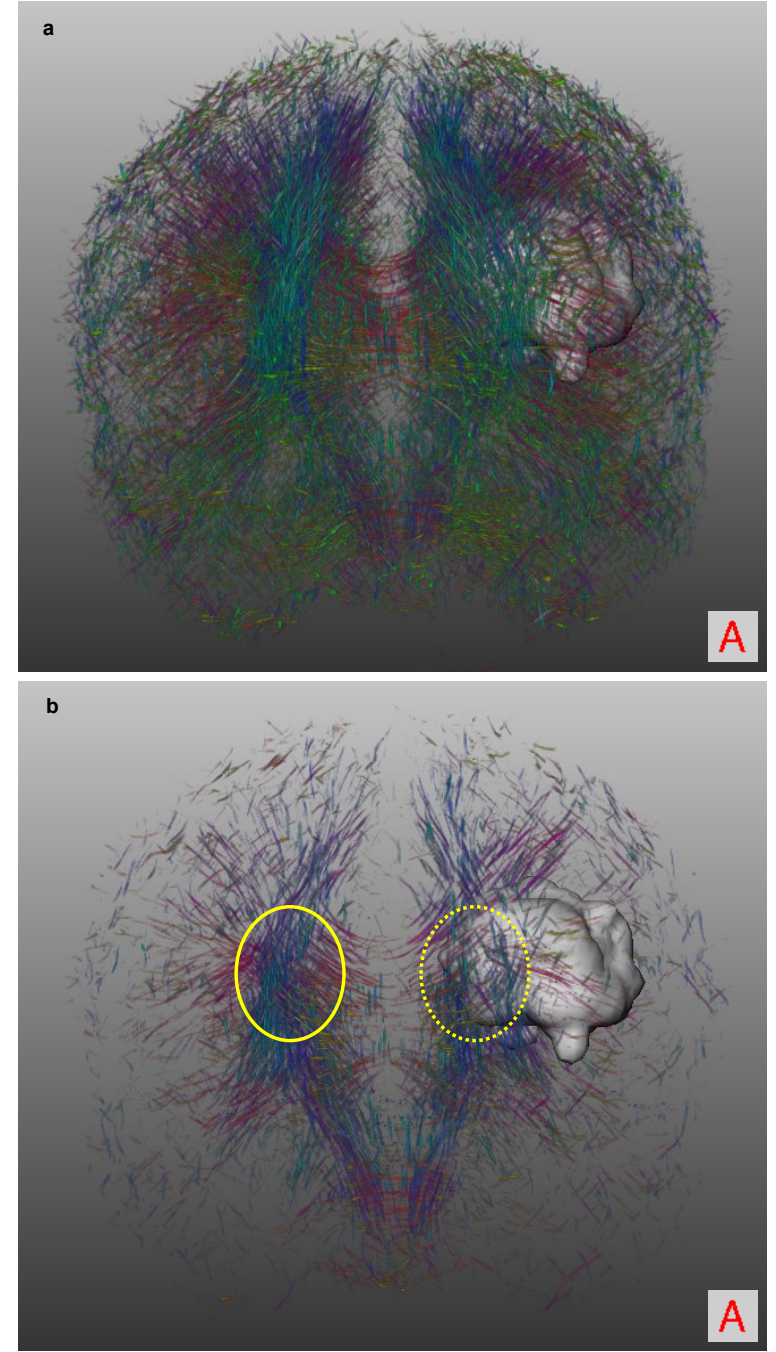

Figure 9. Coronary view of volume rendering and anatomy-driven visualization with focus on left and right CST from Patient B. (a) Volume rendering with integrated tumor surface in the left hemisphere. (b) Anatomy-focused Volume

LIC that reveals a reduced fiber density of the left branch of the CST (dotted yellow ellipse) in comparison to the right branch (yellow ellipse).

Figure 10 shows a final comparison of four different methods to visualize the $\mathrm{CC}$ of Patient $\mathrm{A}$ in an axial view. In contrast to previous axial representations, a view from bottom to top is given. In Figure 10a, showing a volume rendering of the LIC volume, the $\mathrm{CC}$ is occluded by fibers running from anterior to posterior (depicted in green) and from superior to inferior (depicted in blue). A disruption of the $\mathrm{CC}$ as shown in Figure $7 \mathrm{~b}$ cannot be seen. Figure 10b shows a volume rendering with an optimally adjusted lookup table and transparency to present the $\mathrm{CC}$ in more detail. Since in LIC data single fibers do not differ enough in their intensity the application of transfer functions is of limited effect. Especially fibers from the CC and the CST are of similar intensity in this example, which leads to the $\mathrm{CC}$ still being occluded and the disrupted area of the $\mathrm{CC}$ can just be seen to some extent (marked by yellow arrows). Furthermore, the adjustment in the look-up table and the transparency causes skips in the $\mathrm{CC}$ (marked by dotted yellow ellipse). Figure 10c shows a volume rendering with applied clipping to select the CC. Since the course of the $\mathrm{CC}$ is selectable by planar clipping planes the disruption becomes visible to some extent (marked yellow arrows) but is still partially occluded. With more curved fiber bundles, planar clipping planes would not be sufficient and curved planes would have to be used. Furthermore, the anatomical context of the clipped region might be missing. Figure $10 \mathrm{~d}$ shows the anatomy-focused Volume LIC with emphasized $\mathrm{CC}$ and transparent rendering of fibers running along the viewing axis. Here, the disrupted area of the $\mathrm{CC}$ with reduced fiber density becomes visible (marked by yellow arrows). This method is applicable to more curved fiber bundles as well, but its accuracy depends on the used anatomical atlas and on the precision of the matching between atlas and individual patient dataset.

\section{DISCUSSION and CONCLUSION}

With anatomy-focused Volume LIC for brain white matter visualization, we have presented a novel approach to utilize Volume LIC for dwMRI data. To minimize occlusion effects, an integration of an anatomical atlas is proposed, that is registered to the individual dwMRI dataset. While generating the anisotropic glyph sample input texture for the LIC algorithm, the atlas can be used to vary the sample placement probability in different anatomic regions. Anatomy-focused Volume LIC makes it possible to get an insight into the inner structures of the brain and to highlight single fiber bundles. With an adaptation of the orientation-dependent transparency rendering approach, fibers that run along the viewing axis can be rendered transparent to clarify the display even more. The presented results focus on two patient datasets with different types and locations of brain tumors. Next steps will be a broader clinical evaluation with more patient datasets with different pathologies. Furthermore, it has to be examined how well a registered anatomical atlas fits an individual patient dataset, especially when brain structures are considerably displaced by pathologies. Here, novel techniques of elastic atlas matching have to be evaluated in order to avoid manual adjustments. Additionally, other anatomical atlases with different segmentations of anatomic structures will be analyzed as to their potential use for generating atlas-based input structures to the LIC algorithm. 

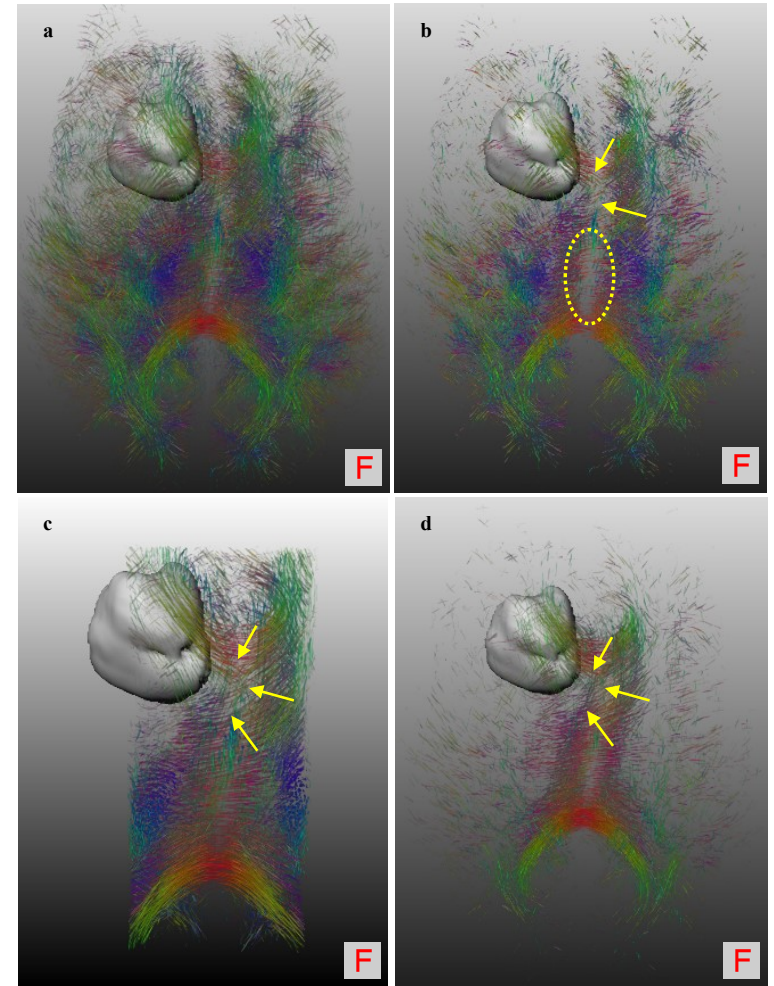

Figure 10. Comparison of the depiction of the $\mathrm{CC}$ from Patient $A$ on (a) volume rendering, (b) volume rendering with adjusted look-up table and transparency, (c) volume rendering with applied clipping and (d) anatomy-focused Volume LIC with emphasized $\mathrm{CC}$ and transparent fibers running from superior to inferior. The tumor surface in the left hemisphere is integrated in all representations. Yellow arrows mark the region where the $\mathrm{CC}$ is disrupted. The dotted yellow ellipse marks a region with skips in the $\mathrm{CC}$ due to look-up table and transparency adjustments.

\section{REFERENCES}

[AMBS13] AUZINGER, THOMAS; MistelbaUER, GABRIEL; BACLIJA, IVAN; SCHERNTHANER, RUDIGER; KOCHL, ARNOLD; WIMMER, MICHAEL; GROLLER, M. EDUARD; BRUCKNER, STEFAN: Vessel visualization using curved surface reformation. In: IEEE Transactions on Visualization and Computer Graphics Bd. 19 (2013), S. 2858-2867

[BHWV07] BURNS, MICHAEL; HAIDACHER, MARTIN; WEIN, WOLFGANG; VIOLA, IVAN; GRÖLLER, M EDUARD: Feature Emphasis and Contextual Cutaways for Multimodal Medical Visualization. In: Eurographics / IEEE VGTC Symposium on Visualization 2007 : Eurographics Association, 2007, S. $275-282$
[CaLe93] CABRAL, BRIAN; LEEDOM, LEITH CASEY: Imaging vector fields using line integral convolution. In: Proceedings of the 20th annual conference on Computer graphics and interactive techniques SIGGRAPH '93. Bd. 27. New York, New York, USA : ACM Press, 1993 — ISBN 0897916018, S. 263-270

[ChFS03] CHEN, LI; FUJISHIRO, ISSEI; SUZUKI, YASUKO: Comprehensible Volume LIC Rendering based on 3D Significance Map. In: Visualization and Data Analysis 2002. Bd. 4665, 2003, S. 142-153

[ChRM94] Christensen, G. E.; RABBitT, R. D.; MILLER, M. I.: 3D brain mapping using a deformable neuroanatomy. In: Physics in Medicine and Biology Bd. 39 (1994), Nr. 3, S. $609-618$

[Comp18] Computational Morphometry Toolkit (CMTK). URL

https://www.nitrc.org/projects/cmtk/. abgerufen am 2018-12-17

[HeAn04] HELGELAND, ANDERS; ANDREASSEN, OYVIND: Visualization of vector fields using seed LIC and volume rendering. In: IEEE Transactions on Visualization and Computer Graphics Bd. 10 (2004), S. 673-682

[HESK17] HÖLLER, M; EHRICKE, H.-H.; SYNOFZIK, M; KLOSE, U; GROESCHEL, S: Clinical Application of Fiber Visualization with LIC Maps Using Multidirectional Anisotropic Glyph Samples (A-Glyph LIC). In: Clinical Neuroradiology Bd. 27 (2017), Nr. 3, S. 263-273

[HOKG14] HÖLLER, MARK; OTTO, KAY-M.; Klose, Uwe; GROESCHEL, SAMUEL; EHRICKE, HANS-H.: Fiber Visualization with LIC Maps Using Multidirectional Anisotropic Glyph Samples. In: International Journal of Biomedical Imaging Bd. 2014 (2014), S. 1-14 - ISBN 1687-4188 (Print) 1687-4188 (Linking)

[InGr97] INTERRANTE, V.; GROSCH, C.: Strategies for effectively visualizing $3 \mathrm{D}$ flow with volume LIC. In: roceedings of the 8 th Conference on Visualization '97 : IEEE Computer Society Press, 1997, S. $421 \mathrm{ff}$.

[KRRS09] KAINZ, BERNHARD; REITER, URSULA; REITER, GERT; SCHMALSTIEG, DIETER: In vivo interactive visualization of four-dimensional blood flow patterns. In: The Visual Computer Bd. 25 (2009), Nr. 9, S. 853-862 
[LHDV04] LARAMEE, ROBERT S.; HAUSER, Helwig; Doleisch, Helmut; VROLIJK, BENJAMIN; POST, FRITS H.; WEISKOPF, DANIEL: The State of the Art in Flow Visualization: Dense and Texture-Based Techniques. In: Computer Graphics Forum Bd. 23 (2004), Nr. 2, S. 203-221 - ISBN 1467-8659

[LiMo05] LIU, ZHANPING; MOORHEAD, ROBERT J.: A texture-based hardwareindependent technique for time-varying volume flow visualization. In: Journal of Visualization (2005), S. 235-244

[MCAG06] Miller, M. I.; Christensen, G. E.; AMIT, Y.; GRENANDER, U.: Mathematical textbook of deformable neuroanatomies. In: Proceedings of the National Academy of Sciences Bd. 90 (2006), Nr. 24, S. 1194411948

[Mevi18] MeVis Medical Solutions AG: MeVisLab. URL https://www.mevislab.de. abgerufen am 2018-10-04

[MOJJ08] MORI, SUSUMU; OISHI, KENICHI; JIANG, HANGYI; JIANG, LI; LI, XIN; AKHTER, KAZI; HuA, KEGANG; FARIA, ANDREIA V.; U. A.: Stereotaxic white matter atlas based on diffusion tensor imaging in an ICBM template. In: NeuroImage Bd. 40 (2008), S. 570-582 - ISBN 1053-8119 (Print) 1053-8119 (Linking)

[PaPi99] PAJEVIC, SINISA; PIERPAOLI, CARLO: Color schemes to represent the orientation of anisotropic tissues from diffusion tensor data: Application to white matter fiber tract mapping in the human brain. In: Magnetic Resonance in Medicine Bd. 42 (1999), Nr. 3, S. 526-540

[PeVR09] PEETERS, T. H. J. M.; VILANOVA, A.; ROMENY, B. M. TER HAAR: Interactive Fibre Structure Visualization of the Heart. In: Computer Graphics Forum Bd. 28 (2009), Nr. 8, S. 2140-2150

[RHTE99] REZK-SALAMA, C; HASTREITER, P.; TEITZEL, C.; ERTL, T.: Interactive exploration of volume line integral convolution based on 3D-texture mapping. In: Proceedings Visualization '99 (Cat. No.99CB37067) : IEEE, 1999 — ISBN 07803-5897-X, S. 233-528
[RoMa05] ROHLFING, TORSTEN; MAURER, CALVIN R.: Multi-classifier framework for atlas-based image segmentation. In: Pattern Recognition Letters Bd. 26 (2005), Nr. 13, S. 2070-2079

[SFCN03] SuZUKI, Y.; FUJISHIRO, I.; CHEN, L.; NAKAMURA, H.: Case study: hardwareaccelerated selective LIC volume rendering. In: , 2003

[SHSK10] SCHURADE, R; HLAWITSCHKA, M; SCHEUERMANN, B HAMANN G; KNÖSCHE, T $\mathrm{R}$; ANWANDER, A: Visualizing white matter fiber tracts with optimally fitted curved dissection surfaces. In: Proceedings of Eurographics Workshop on Visual Computing for Biology and Medicine, 2010, S. $41-48$

[SRBG10] SIKACHEV, P.; RAUTEK, P.; BRUCKNER, S.; GRÖLLER, M.E.: Dynamic Focus+Context for Volume Rendering. In: Proceedings of Vision, Modeling, and Visualization 2010, 2010

— ISBN 9783905673791, S. 331-338

[TCGC04] TOURNIER, J D; CALAMANTE, F; GADIAN, D G; CONNELly, A: Direct estimation of the fiber orientation density function from diffusion-weighted MRI data using spherical deconvolution. In: Neuroimage Bd. 23 (2004), Nr. 3, S. 1176 1185 - ISBN 1053-8119 (Print)1053-8119 (Linking)

[TCSV15] TAX, CHANTAL M. W.; CHAMBERLAND, MAXIME; VAN STRALEN, MARIJN; VIERGEVER, MAX A.; WhitTINGSTALl, KEVIN; FORTIN, DAVID; DESCOTEAUX, MAXIME; LEEMANS, ALEXANDER: Seeing More by Showing Less: Orientation-Dependent Transparency Rendering for Fiber Tractography Visualization. In: HodAIE, M. (Hrsg.) PLOS ONE Bd. 10 (2015), Nr. 10, S. e0139434

[ToCC07] TOURNIER, J D; CALAMANTE, F; CONNELLY, A: Robust determination of the fibre orientation distribution in diffusion MRI: non-negativity constrained superresolved spherical deconvolution. In: Neuroimage Bd. 35 (2007), Nr. 4, S. 1459 1472 - ISBN 1053-8119 (Print)1053-8119 (Linking) 